\title{
A COVID-19 model with a nonlinear incidence rate and intervention exit strategies for Kenya
}

Rachel Waema Mbogo ( $\square$ rmbogo@strathmore.edu )

Strathmore University https://orcid.org/0000-0003-1639-1360

\section{Farai Nyabadza}

University of Johannesburg https://orcid.org/0000-0003-3468-5581

\section{Research Article}

Keywords: COVID-19, SLIHRD-model, Non-linear incidence rate, Exit strategies, Behavior change, Mass testing

Posted Date: February 12th, 2021

DOI: https://doi.org/10.21203/rs.3.rs-114213/v2

License: (1) This work is licensed under a Creative Commons Attribution 4.0 International License. Read Full License 


\title{
A COVID-19 model with a nonlinear incidence rate and intervention exit strategies for Kenya
}

\author{
Rachel Waema Mbogo ${ }^{1 *}$, Farai Nyabadza ${ }^{2}$ \\ 1 Institute of Mathematical Sciences, \\ Strathmore University, Nairobi - KENYA. \\ 2 Department of Mathematics and Applied Mathematics, \\ University of Johannesburg, South Africa.
}

\begin{abstract}
The coronavirus disease (COVID-19) is a novel infection caused by SARS-CoV-2, a corona virus type that has previously not been seen in humans. The speedy spread of COVID-19 globally has greatly affected the socio-economic environments and health systems. To effectively address this rapid spread, it is imperative to have a clear understanding of the COVID-19 transmission dynamics. In this study we evaluate a COVID-19 epidemic model with a nonlinear incidence function and a saturating. We propose an $S L I H R D$ data driven COVID 19 model which incorporates individual self initiated behavior change of the susceptible individuals. The proposed model allows the evaluation of the impact of easing intervention measures at specific times. To estimate the model parameters, the model was fitted to the daily reported COVID-19 cases in Kenya. Self initiated behavioral responses by individuals and large scale persistent testing proved to be the most effective measures to flatten the epidemic infection curve.The model illustrates the effect of mass testing on COVID-19 as well as individual self initiated behavioral change when the number of infected individuals increases. The results have significant impact on the management of COVID-19 and implementation of prevention policies.
\end{abstract}

Keywords: : COVID-19; SLIH RD-model; Non-linear incidence rate; Exit strategies; Behavior change; Mass testing

\section{Introduction}

The corona virus 2019, which first started in the Wuhan city of China in December 2019 has greatly affected the socio-economic environment and health systems worldwide. The disease has killed many, altered the definition of normalcy and collapsed economies since it was first reported. The global situation for COVID-19 as at November 18, 2020 stood at over 56 million confirmed cases with the total number of deaths standing at over 1.3 million according to World Health Organization (WHO) [34]. In the mean time, the number of COVID-19 infections in Africa stood at over 1.4 million confirmed cases. In Kenya with a population of about 47 million people, the number of confirmed cases as at November stood at over 71 thousand and over 1300 deaths [34]. The WHO, in February 2020 declared coronavirus disease COVID-19, a global pandemic [35]. The disease poses a huge threat to the global populations $[16,31]$.

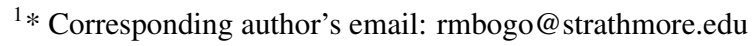


Severe acute respiratory syndrome coronaviruses (SARS-CoV) are enveloped single-stranded RNA viruses that have not been identified in humans before [1]. The SARS-CoV-2 virus affects different people in different ways and spreads mainly from person-to-person who are in close contact. Most patients develop mild illness and recover without hospitalization. Infected persons are assumed to be most infectious when they are symptomatic, but there are reports of individuals transmitting this new coronavirus before they exhibit symptoms, see [36, 37]. Like many respiratory diseases, COVID-19 spreads through aerosols from coughs and sneezes of infected persons. It can also spread surfaces touched by infectious individuals after they have sneezed or coughed into their hands [33, 37]. The most common symptoms for SARS-COV-2 are fever, tiredness and a dry cough. According to the WHO, about $20 \%$ of people who get infected with COVID-19 experience mild symptoms and recover without needing any special treatment [40]. Recovery depends on the strength of the immune system of the infected person. The elderly and people with other underlying medical conditions are at a higher risk of developing severe illness from Covid-19 [10, 17].

On March 15, 2020, the Kenyan Government moved promptly to employ containment measures to contain the rise in COVID-19 infections. Kenya's early implementation of the non-pharmaceutical interventions (NPIs) measures proved to be effective in suppressing the spread of COVID-19. These NPIs included closure of international borders, schools, restaurants, bars and nightclubs, use of face masks in public, banning meetings and social gathering and imposing a dusk to dawn curfew and movement restrictions in the two major county cities, Nairobi and Mombasa, considered as epicenters of Covid-19 infections at the beginning of the epidemic [6,22-24]. The combination of these interventions has had a substantial impact in limiting the community transmission of COVID-19. Another containment strategy the Kenyan Government employed was contact tracing and mass testing.

During the early stages of an emerging infectious disease outbreak, it is important to understand its transmission. Assessment and analysis of transmission dynamics over time can provide insights into the disease transmission and help identify whether control measures are having a quantifiable effect [14]. Analysis of transmission dynamics at early stages can inform predictions about potential future growth, help estimate risk to other counties, and guide the design of alternative interventions. Mathematics, has traditionally been used to gain realistic insight into the epidemiology of infectious diseases transmission dynamics [26]. Mathematical models thus play a vital role in evaluating the readiness of any government in fighting infectious diseases while letting policy makers plan for possible public health scenarios before they arise [11]. Since the COVID-19 pandemic started, mathematical modelling has been at the forefront of informing decision-making [18, 21, 27].

A simple mathematical model was used to trace the temporal course of the South Korea Middle East Respiratory Syndrome Coronavirus (MERS-CoV) outbreak [8]. In [28] an age-structured susceptibleexposed-infected-removed $(S E I R)$ model was used to predict the trajectory of the virus outbreak in Wuhan, China. The projections showed that physical distancing measures were most effective in containing the infection. The SEIR model was extended to forecast the COVID-19 epidemic peaks and sizes in China, see [39, 41]. Further, a mathematical model for MERS-CoV transmission dynamics was used to estimate the transmission rates in two periods due to the implementation of intensive interventions [3, 12]. Several Mathematical models have been used to help plan public health interventions since the first time COVID-19 infection was reported [2-4, 7, 9, 20,38]. Other authors used clinical approaches to explain the disease outbreak [31].

As countries begin to relax many of the containment measures initially imposed to control COVID-19, it is important to evaluate the impact of different exit strategies for the disease [27]. Since July 2020, the Kenyan Government relaxed some of the COVID-19 containment measures including opening of 
restaurants and places of worship and the removal of travel restrictions into and out of Mombasa and Nairobi counties [25]. Further from September 2020, a phased return to school was allowed for learning institutions. In this study, we extended the work in [17]. The proposed SLIHRD model employing a nonlinear incidence rate, which explains the effect of mass testing on COVID-19 spread as well as individual self initiated behavioral change when the number of infected individuals increases will be used to analyze the above COVID-19 exit strategies. We also innovatively model a declining recovery rate, that depends on the number of hospitalisation, assuming that as hospitalisations increase, the recovery rate is impacted by the number of individual admissions. As hospital admissions increase, there has been a noted fatigue of health care workers and declining quality of service leading to reduced recovery rates.

The rest of the paper is organized as follows: in Section 2, the model is formulated. In Section 3, numerical simulations including the fitting of the model to COVID-19 data are carried out. The effects of individual behavioral change, intermittent lifting of intervention measures and mass testing are also investigated. In Section 4, we provide discussions and recommendations.

\section{Model Description and Formulation}

\subsection{Model Assumptions}

The study employs an SLIHRD model with the following assumptions:

- The Kenyan population is sufficiently large to take care of the variations in the spread of COVID 19 , so a deterministic model is considered.

- We assumed Kenya to be a closed system with a constant population size of 47 million (that is , $S+L+I_{s}+I_{a}+H+R+D=47$ million) throughout the course of the epidemic see [18].

- Each individual has the same chance of making contact with other individuals- homogeneous mixing.

- The spread of the coronavirus only occurs between humans to humans [18].

- The incubation period of COVID -19 is 14 days.

- Individuals infected by the disease can recover. Current evidence on the pandemic does not suggest that the recovered individuals become susceptible again. This is inline with other respiratory infections such as MERS [30].

- Natural births, natural deaths, and ageing are overlooked. This is largely due to the short modelling time.

We consider a compartmental model in which each compartment variable denotes the number of individuals who belong to one of the following states: susceptible $(S)$, that is, individuals who are not yet infected but are at risk of the infection. When the Susceptible interact with infected individuals they get infected with the virus and move into compartment latent $(L)$. The term $e^{-\gamma \tau}$ is the probability of

an exposed person surviving to being infectious in 4-5 days (latent period). $\frac{1}{\tau}$ is the incubation period that represent the length of time before the infected individuals can become infectious. Those who develop symptoms immediately move to severe infectious class (symptomatic) denoted by compartment $I_{s}$. Those who have their symptoms delayed due to strong immune system move to mild infectious class (asymptomatic) denoted by $I_{a}$. Both individuals in $I_{a}$ and $I_{s}$ are infectious though those in $I_{a}$ have not developed symptoms, see $[18,19]$. The asymptomatic and symptomatic individuals can be recognized 
through contact tracing and mass testing and isolated (taken to hospital /isolation Centres) denoted compartment $(H)$. The isolated infected individuals (those in hospitals or isolation centres) can recover and move to compartment $R$ or succumb to COVID-19 and move to compartment (D) [18].

The total population at any time $t$, is denoted by $N(t)$ and is given by

$$
N(t)=S(t)+L(t)+I_{s}(t)+I_{a}(t)+H(t)+R(t)+D(t) .
$$

The force of infection is modelled by $\lambda(t)$ and given by the nonlinear incidence rate:

$$
\lambda(t)=\frac{\beta_{1} I_{a}(t)+\beta_{2} I_{s}+\beta_{3} H(t)}{1+\eta\left(I_{s}(t)+H(t)\right)},
$$

where $\beta_{1}, \beta_{2}$ and $\beta_{3}$ are the effective contact rates between susceptibles and asymptomatic infected individuals, susceptibles and symptomatic infected individuals and susceptibles and hospitalized individuals respectively. The nonlinear incidence rate $\lambda(t)$ is more reasonable than the most used bi-linear incidence rate. The expression $\frac{1}{1+\eta\left(I_{s}(t)+H(t)\right)}$ measures the behavioral change of individuals when the number of infected and hospitalised individuals increases. The parameter $\eta$ is the percentage of total effort required by an individual to contain/mitigate the COVID-19 epidemic spread resulting from the behavioral change in the population leading to adherence to COVID-19 preventive measures, see for instance [15].

When the susceptible are infected, they move into compartment $L$, referred to as latent or exposed. Here we assume a fraction $\epsilon \in(0 ; 1)$ of the general population are locked down / self-quarantined and only the remaining fraction $1-\epsilon$ of the population are interacting in non-household settings such as work and market places. An exposed/latent individual undergoes an average incubation period of $\frac{1}{\tau}$ that represents the length of time before the infected individuals can become infectious, see also [13]. The term $e^{-\gamma \tau}$ is the probability of an exposed person surviving to become infectious in 4-5 days (i.e the latent period). The parameter $\gamma$ is the rate at which the exposed become infectious. To evaluate the impact of contact tracing for the detection of new COVID-19 patients, we divide the infected class into two, those who have their symptoms delayed due to strong immune system (asymptomatic) $I_{a}$ and those who develop symptoms immediately (symptomatic) $I_{s}$. Both individuals in $I_{s}$ and $I_{a}$ are infectious though those in $I_{a}$ may have not developed symptoms. The symptomatic individuals can be identified and either taken to hospital/isolation centers before recovery at a rate $\alpha_{1}$ or die due to the disease at a rate $\mu_{s}$. Hospitalised/isolated individuals can either recover or they die due to the disease. Infected individuals without symptoms,some can recover from the disease depending on ones immunity, develop symptoms and move to symptomatic class or others are traced,screened and isolated in hospitals or isolation centres. $\alpha_{2}$ is the disease progression rate of the asymptomatic individuals. A proportion $\rho$ of asymptomatic individual who progress get identified and isolated immediately at the rate $\alpha_{2} \rho$, while a proportion $1-\rho$ of those who progress become symptomatic without isolation at rate $(1-\rho) \alpha_{2}$. The asymptomatic individuals recover at a rate $\kappa$. The symptomatic infectives are hospitalised at a rate $\alpha_{1}$.

We assume that, a hospitalized/isolated individual moves to the recovered compartment $R$ at a rate modelled by the nonlinear treatment saturation function $\frac{\omega H(t)}{1+\zeta H}$, where $\zeta$ is the treatment delay parameter and $\omega$ is the recovery rate. The function $\frac{1}{1+\zeta H}$ is a measure of the recovery inhibition due to increased hospitalized/isolated individuals who may be too many to receive the necessary medical attention given the limited available health care resources. Hospitalised individuals die at a rate $\mu_{h}$.

The flow of COVID-19 infection is given in Figure 1. 


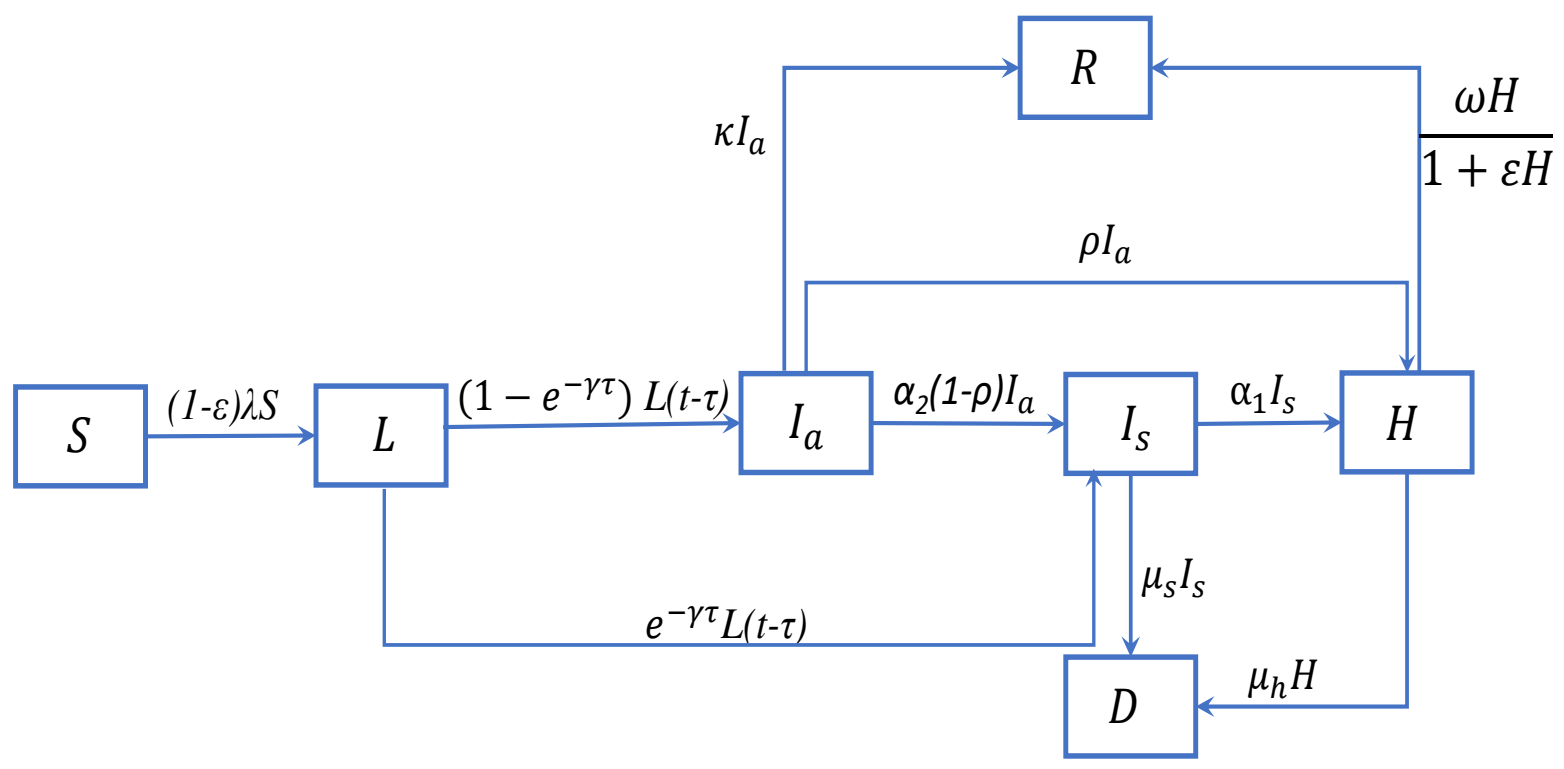

Figure 1: The model diagram

Given the flow diagram in Figure 1, the model description we have the following system of non-linear ordinary differential equations:

$$
\begin{aligned}
\frac{d S(t)}{d t} & =-(1-\epsilon) \lambda(t) S(t) \\
\frac{d L(t)}{d t} & =(1-\epsilon) \lambda(t) S(t)-\gamma L(t) \\
\frac{d I_{s}(t)}{d t} & =e^{-\gamma \tau} L(t-\tau)+\alpha_{2}(1-\rho) I_{a}(t)-\alpha_{1} I_{s}(t)-\mu_{s} I_{s}(t), \\
\frac{d I_{a}(t)}{d t} & =\left(1-e^{-\gamma \tau}\right) L(t-\tau)-\alpha_{2} I_{a}(t)-\kappa I_{a}(t), \\
\frac{d H(t)}{d t} & =\alpha_{1} I_{s}(t)+\alpha_{2} \rho I_{a}(t)-\frac{\omega H(t)}{1+\zeta H(t)}-\mu_{h} H(t), \\
\frac{d R(t)}{d t} & =\kappa I_{a}(t)+\frac{\omega H(t)}{1+\zeta H(t)}, \\
\frac{d D(t)}{d t} & =\mu_{h} H(t)+\mu_{s} I_{s}(t),
\end{aligned}
$$

subject to the following initial conditions

$S(0)>0, L(0) \geq 0, I_{s}(0) \geq 0, I_{a}(0) \geq 0, H(0) \geq 0, R(0) \geq 0, D(0) \geq 0$. 


\subsection{Positivity and Boundedness of solution}

All solutions of system (2) remain positive and bounded for all the time. For detailed prove please see $[19,29]$.

\subsection{Equilibria analysis of the model}

The basic reproduction number $R_{0}$, is defined as the number of secondary infections produced by one infective that is introduced into an entirely susceptible population at the disease free equilibrium [5]. The next generation matrix approach is frequently used to compute $R_{0}$, see [32] for some detailed explanation.

System (2) has a disease-free equilibrium (DFE) given by

$$
E_{0}=\left(S_{0}, 0,0,0,0,0,0\right) .
$$

The model basic reproduction number, $R_{0}$, which is determined as the spectral radius of $F V^{-1}$ (the next generation matrix), is given by

$$
R_{0}=R_{I a}+R_{I s}+R_{H}
$$

where

$$
\begin{aligned}
& R_{I a}=\frac{\beta_{1}(1-\epsilon)\left(1-e^{-\gamma \tau}\right)}{\gamma\left(\alpha_{2}+\kappa\right)}, \\
& R_{I s}=\frac{\beta_{2}(1-\epsilon) e^{-\gamma \tau}\left(\kappa+\alpha_{2} e^{\gamma \tau}(1-\rho)+\rho \alpha_{2}\right)}{\gamma\left(\alpha_{1}+\mu_{s}\right)\left(\kappa+\alpha_{2}\right)}, \\
& R_{H}=\frac{\beta_{3}(1-\epsilon) \rho \alpha_{2}\left(1-e^{-\gamma \tau}\right)}{\gamma\left(\alpha_{2}+\kappa\right)\left(\mu_{h}+\omega\right)} .
\end{aligned}
$$

Here $R_{0}$ is the sum of three terms each representing the average new infections contributed by each of the three infectious classes. $R_{I a}$ represents the new cases generated by infected asymptomatic individuals in the community represented by compartment $I_{a}, R_{I s}$ represents the new cases generated by infected symptomatic individuals represented by compartment $I_{s}$ and $R_{H}$ represents new cases generated by patients hospitalized or quarantined (In isolation centres).

From $R_{0}$ in equation (3), each term is multiplied by $\beta(1-\epsilon) e^{-\gamma \tau}$ which represents the effects of adherence to the government control measures especially adherence to social or physical distancing, partial lockdown measures, personal hygiene and individual immune response. Therefore, if social distancing is kept, or lockdown measures are maintained, then the emergency of new corona cases is reduced. Therefore the government's campaigns of "maintenance of social distance", "prolonging lockdown" and "personal hygiene" are critical in preventing the development of new cases.

It is therefore evident from $R_{0}$ that an increase in adherence to government measures and aggressive mass testing would reduce the severity of COVID-19 in Kenya. To improve the mass testing exercise, the government should invest in effective testing kits and large scale aggressive testing. 
From Theorem 2 in [32], we have the following result:

Theorem 1. The DFE, $E_{0}$ of model (2) is locally asymptotically stable when $R_{0}<1$ and unstable if $R_{0}>1$. However if $R_{0}=1$, each index case generates one new infectious case, hence the disease persists in a stable state but there will be no epidemic.

\section{Numerical Simulations}

In this section, we present a series of numerical results of system (2) using COVID-19 reported cases data in Kenya to predict and estimate the incidence of the virus in the country considering the laid down intervention measures and also evaluate the impact of lifting intervention policies at specific times.

\subsection{Application of the model to COVID-19 data in Kenya}

COVID-19 cases in Kenya were collected from March 13, 2020 when the first imported case was registered to October 15, 2020 (31 weeks since the cases were reported in Kenya) from the Ministry of Health $(\mathrm{MoH})$ as shown in Figure 2.

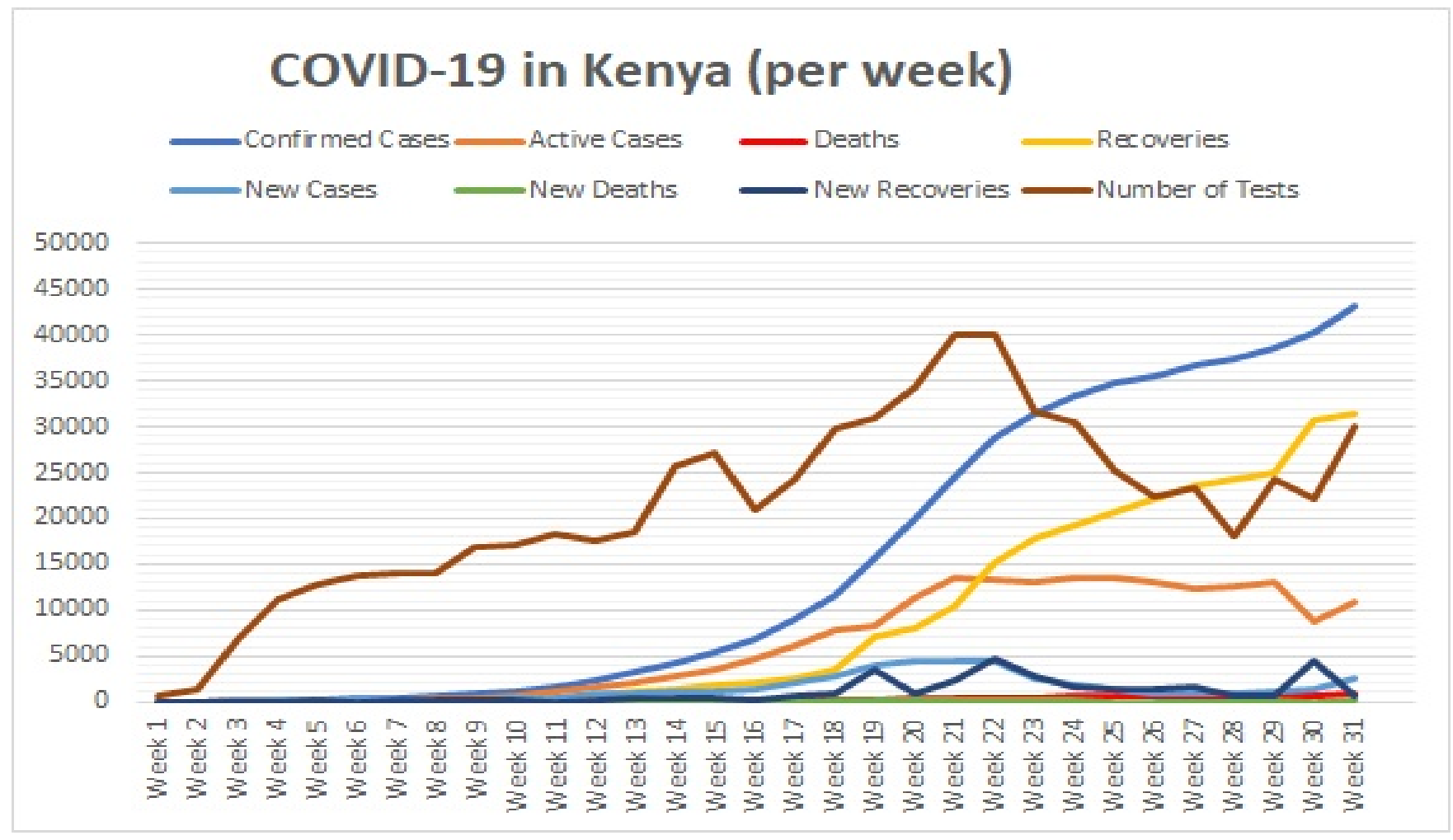

Figure 2: Weekly COVID-19 data for Kenya (March 13-October 15, 2020), for confirmed cases, active cases, deaths, recoveries, new cases, new deaths, new recoveries and the number of test done.

\subsection{Fitting model to data}

Using least squares curve fitting method, the model system (2) was fitted to the COVID-19 reported cumulative cases in Figure 2 and the number of reported deaths (from March 13 to July 2020, before the Kenyan Government eased some of the disease control measures) as shown in Figures $3 \mathrm{a}$ and $3 \mathrm{~b}$ and model parameters values resulting from the fitting process are 
$\beta_{1}=0.2607, \beta_{2}=0.4605, \beta_{3}=0.001, \kappa=0.0115, \alpha_{1}=0.133, \alpha_{2}=0.072, \gamma=0.08$,

$\omega=0.01169, \epsilon=0.4533, \rho=0.62, \eta=0.0007, \mu_{s}=0.0901, \mu_{h}=0.032, \zeta=0.1003$ and $\tau=0.115$.

The initial conditions used are

$S(0)=4.7 \times 10^{7}, E(0)=10, I_{a}(0)=1, I_{m}(0)=1, H(0)=0, Q(0)=0, R(0)=0, D(0)=0$.

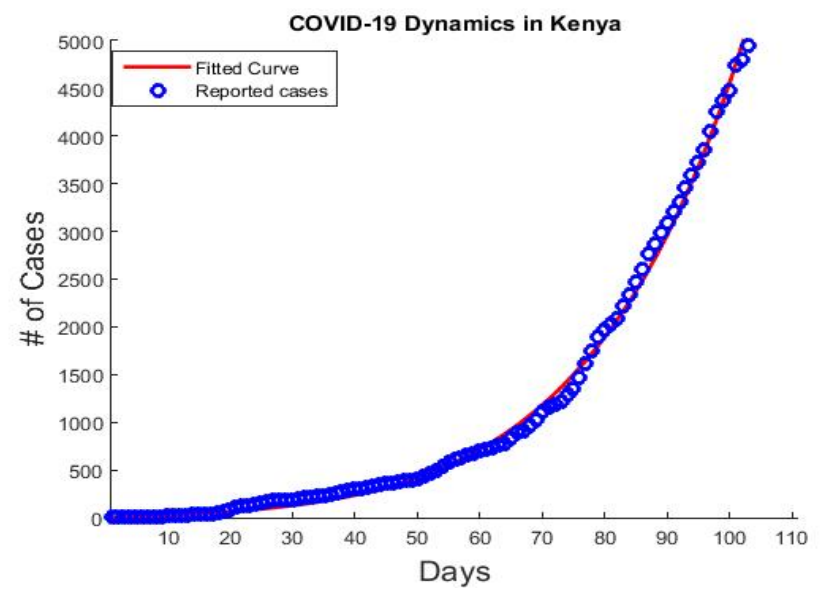

(a) COVID-19 cases with actual Kenyan Data

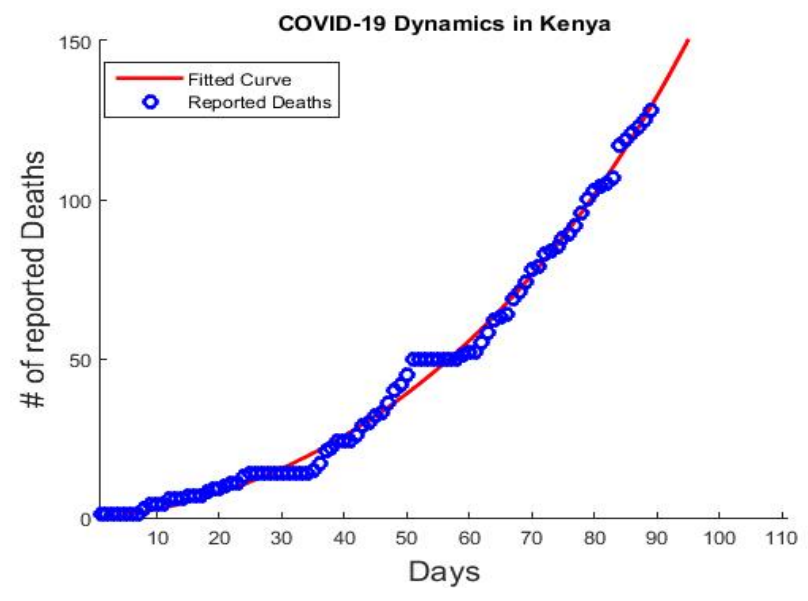

(b) COVID-19 Deaths with actual Kenyan Data

Figure 3: Curve fitting with reported Kenyan data

The model is used to first reproduce the observed trajectory of COVID-19 in Kenya. The model reasonably mimics the observed data, that is the reported cases in Kenya and the number of deaths. Hence the model can be used to make projections of the likely course of the disease as shown in Figures $3 \mathrm{a}$ and $3 \mathrm{~b}$.

\subsection{Model projections}

Using the parameter values and initial conditions, the projected curves for the cumulative cases and number of deaths are displayed in Figures $4 \mathrm{a}$ and $4 \mathrm{~b}$. 


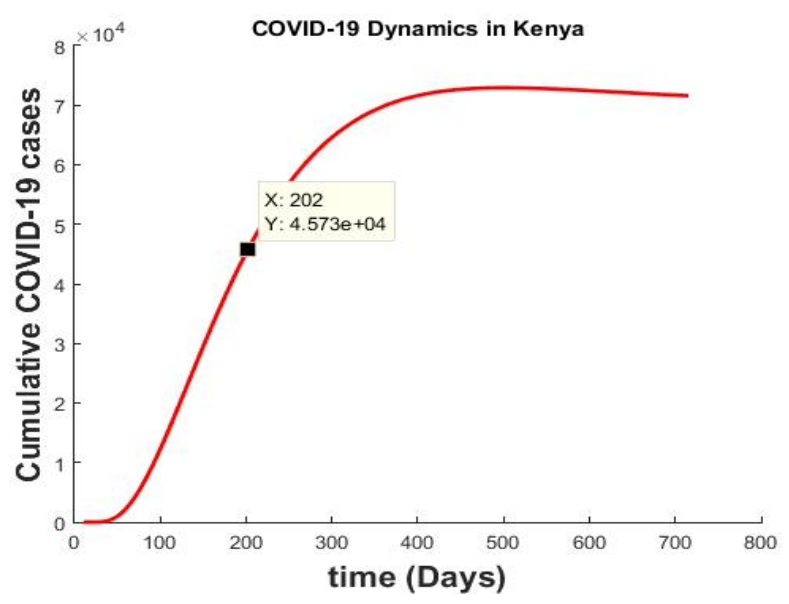

(a) COVID-19 projected cases in Kenya

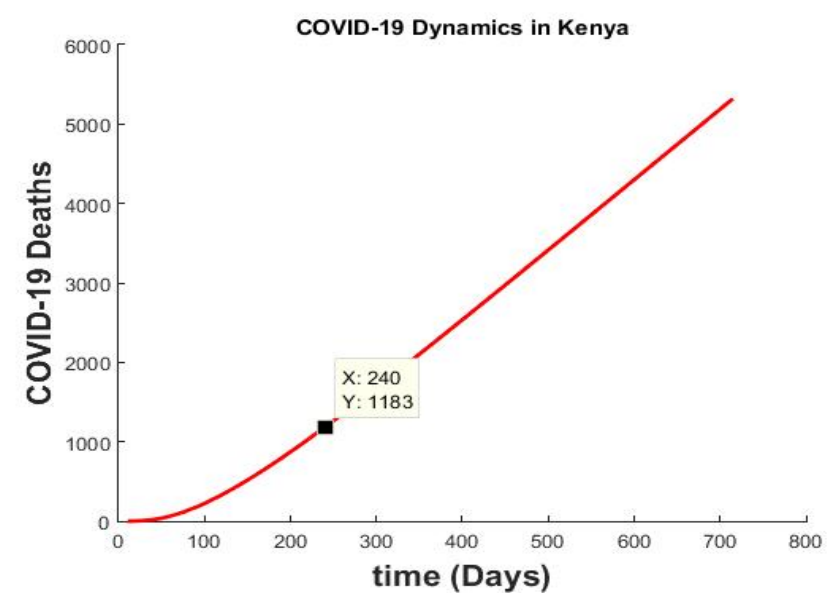

(b) COVID-19 projected Deaths in Kenya

Figure 4: Projections of COVID-19 Cumulative cases and number of deaths Kenya over a two year period

Figure 4a shows that if the situation was to remain as of July 2020, before some intervention measures were lifted, then by mid October 2020 the cumulative COVID-19 cases would be above 45000 (the marked point on the graph).

The graph in Figure 4b shows that by mid November 2020, the number of deaths induced by COVID-19 would be around 1185 (see the marked point). To flatten the COVID-19 cumulative cases curve, the Kenyan government should therefore strictly deploy and implement existing control measures against COVID-19 in every county.

\subsection{Impact of relaxing the government imposed social distancing measures on COVID- 19 spread}

In order to decelerate the spread of SARS-CoV-2, the Kenyan government imposed strict physical distancing measures (closure of international borders, schools, restaurants, bars and nightclubs, use of face masks in public, banning meetings and social gathering and imposing a dusk to dawn curfew and movement restrictions in the two major city counties) since March 20, 2020. As evidence is emerging that these measures may have slowed the spread of the pandemic, it is important to assess the impact of any changes in strategy, including scenarios for the broader relaxation of social distancing and other measures. Attention is now turning to how interventions can be lifted while continuing to restrict transmission. Forecasting the impact of different likely COVID-19 exit strategies is the current central challenge requiring mathematical modeling, but many uncertainties remain.

We simulate a range of different strategies for lifting COVID-19 intervention measures with a societywide relaxation of lock-down measures and in the presence of different non-pharmaceutical interventions, to estimate the number of new infections. 


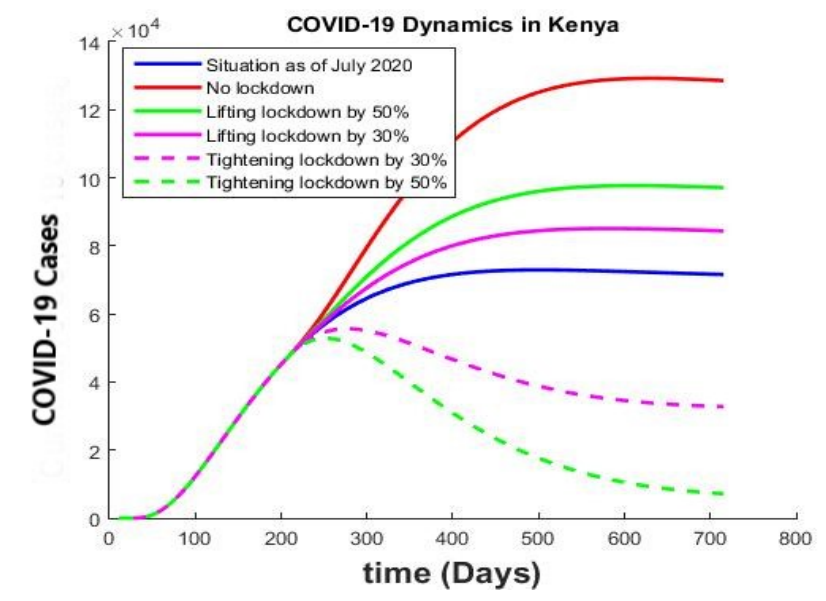

(a) COVID-19 dynamics with lifting of lock-down intervention measures

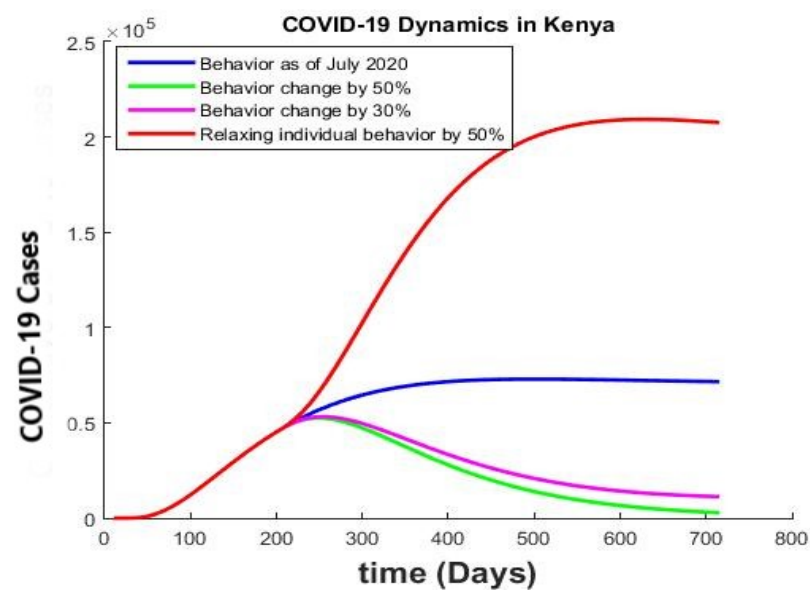

(b) COVID-19 dynamics with individual behavior change

Figure 5: Impact of individual behaviour change and lock-down lifting on cumulative COVID-19 cases.

Figure 5a shows the impact of the partial lifting of the government control measures on the disease transmission dynamics. The simulations show that if the government opened the economy fully in mid October 2020, the cumulative cases would level at about 130,000 cases as shown by the red line in Figure 5a. If the government allowed $50 \%$ opening of the economy, i.e (half of the of the people resume work and partially opening schools) then the dynamics of the disease will be as shown by the green line in Figure 5a. If $30 \%$ of the economy is opened in mid October 2020, then the disease dynamics will be as depicted by pink line in Figure 5a. Lastly if the government had tightened further the lock down by $50 \%$ in mid October 2020, then there would be minimal new cases as depicted by the dotted green line in Figure 5a.

We now considered the impact of individual behavior change on the projected cumulative COVID-19 cases. Figure $5 \mathrm{~b}$ gives insights on effects of behaviour change on the disease spread. The blue line in the $5 \mathrm{~b}$ shows the situation as it was in July, 2020. If individuals relax their behavior of observing social distancing and hygiene by $30 \%$, then the increase in cumulative COVID-19 cases will be as shown by the red line in Figure 5b. If individuals change their behavior to observe social distancing and hygiene by $30 \%$, then the cumulative COVID-19 cases will be as shown by the pink line in Figure $5 \mathrm{~b}$. The green line in Figure $5 \mathrm{~b}$ shows the dynamics of the disease when $50 \%$ of individuals adhere to social distancing and hygiene measures from mid October 2020. Therefore if all individuals follow the social distancing and hygiene prevention measures for COVID-19, then there will be decline in new cases as depicted by the dotted green line in Figure $5 \mathrm{~b}$. 


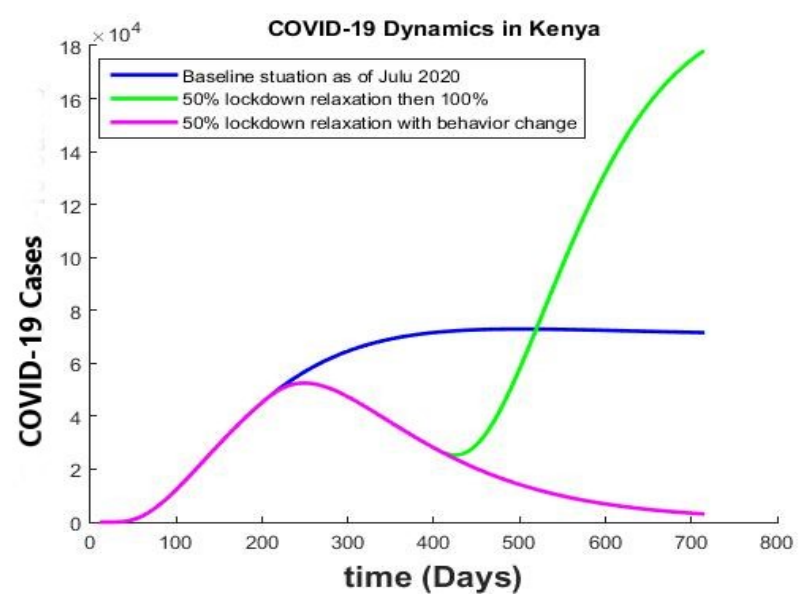

(a) COVID-19 dynamics with lockdown easing and individual behavior

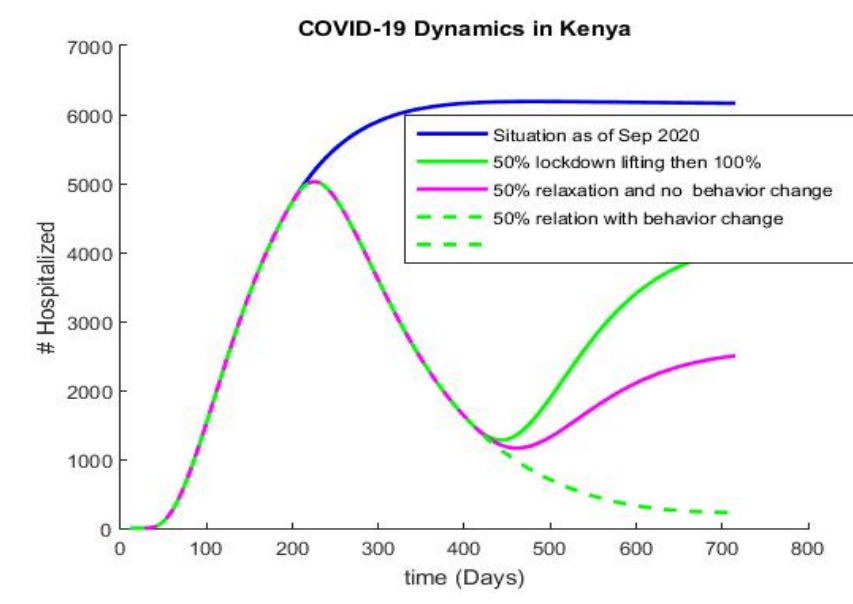

(b) Hospitalized cases with Lock-down easing and individual behavior

Figure 6: Impact of individual behaviour change and lock-down lifting on number of hospitalized cases.

Figure 6a shows the impact of easing the government lock down control measures on the disease transmission dynamics given individual behavior change. The simulation shows that if the government allowed $50 \%$ opening of the economy in mid October 2020 with individual change of behavior, followed by relaxation of the lock down measures to $100 \%$ by mid April 2021, then the dynamics of the disease will be as shown by the green line in Figure 6a. If $50 \%$ relaxation of the lockdown with individual behavior change from October 2020, then there will be a sustained decline of new cases as depicted by the pink line in Figure 6a.

We analyzed the impact of individual behavior change and lock down relaxation on the hospitalized COVID-19 cases. Figure $6 \mathrm{~b}$ gives dynamics of the hospitalized cases given individual behavior change and lifting of the lockdown control measures. The blue line in the $6 \mathrm{~b}$ shows the situation as it was in July, 2020. The simulation shows that If the government allowed $50 \%$ opening of the economy in mid October 2020 with individual change of behavior, followed by relaxation of the lockdown measures to $100 \%$ by mid April 2021, then the number of hospitalized cases will be as depicted by the green line in Figure 6b. If the government allowed $50 \%$ opening of the economy in October 2020 with individual change of behavior, followed by individuals ignoring the social distancing and hygiene measures by mid April 2021, then the number of hospitalized cases will be as depicted by the pink line in Figure 6b. If $50 \%$ relaxation of the lockdown with individual behavior change from October 2020, then there will be a minimal number of hospitalized cases as depicted by the dotted green line in Figure $6 \mathrm{~b}$.

\subsection{Impact of mass testing on COVID-19 spread given relaxation of imposed measures}

We simulate the effect of mass testing considering different reopening strategy scenarios on the nonhospitalized COVID-19 infected populations. 


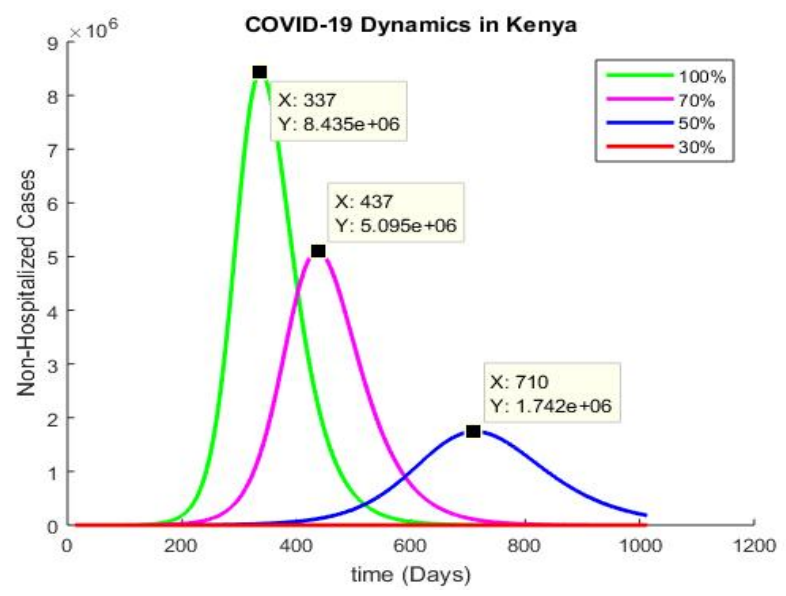

(a) Intervention relaxation impact on Nonhospitalized infected population

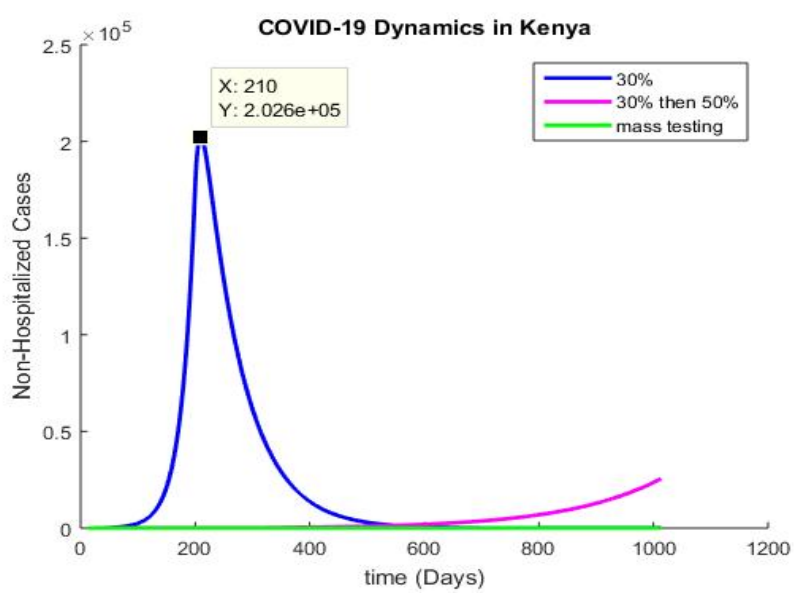

(b) Impact of aggressive mass testing on infected population

Figure 7: Impact of Mass testing and lock-down lifting on COVID-19 diffusion given individual behavior.

Figure 7 shows the impact of a partial lifting of government control measures on the non-hospitalized populations given individual behavior and effective mass testing. The simulation shows that with $100 \%$ opening of the economy in mid October 2020 while ignoring social distancing and personal hygiene, the cases will reach a peak of about 8 million in about 4 months from October as shown by the green line in the graph $7 \mathrm{a}$. If the government allowed $70 \%$ opening of the economy assuming no change in individual behavior then the number of non-hospitalized population will be as shown by the pink line in Figure 7a. If $50 \%$ of the economy is opened with individuals social distancing, then the population of those infected and not yet hospitalized/isolated will be as depicted by blue line in Figure 7a. Lastly if the government initially allowed only $30 \%$ opening of the economy in mid October 2020, then there will be minimal new cases as depicted by the red line in Figure $7 \mathrm{a}$.

Figure $7 \mathrm{~b}$ gives analysis of the impact of partial lifting of government control measures on the nonhospitalized populations given individual behavior and effective mass testing. If the Government only allows $30 \%$ opening of the economy mid October 2020, then Kenya will only be worried of the already infected population as shown by the blue line in Figure 7b. The pink line in Figure 7b shows the dynamics of the disease when $30 \%$ lifting is imposed in mid October 2020 followed by $50 \%$ lifting of the measures in April 2021 while observing social distancing and personal hygiene. The green line in Graph 7b shows the dynamics of the disease when aggressive targeted contact tracing and effective mass testing is applied and the government imposes 30\% lifting in mid October 2020 followed by $50 \%$ lifting of the measures in April 2021 while observing social distancing and personal hygiene. With aggressive targeted contact tracing and mass testing, the asymptomatic and symptomatic patients who are not yet in hospital will be identified and either hospitalized or quarantined in isolation centres hence preventing further transmission. The asymptomatic persons are the problem and the contact tracing and mass testing should be targeting this population. 


\subsection{Effect of relaxing the government imposed social distancing measures on COVID-19 deaths}

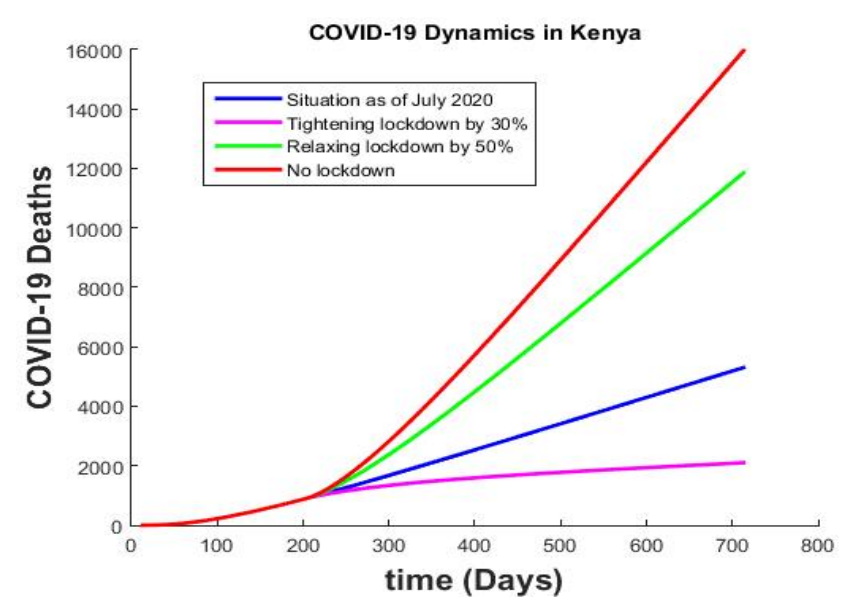

(a) The impact of individual behavior change on COVID-19 deaths

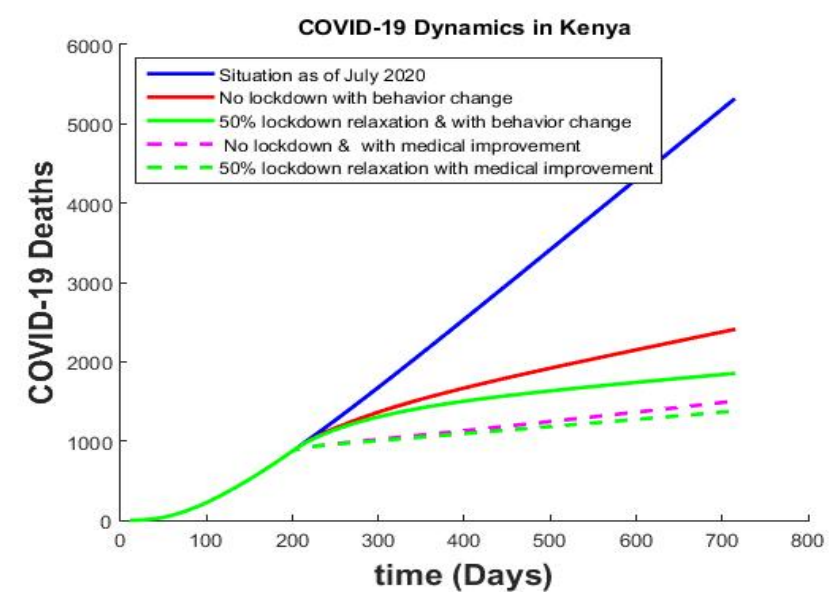

(b) The impact of improved healthcare services on COVID-19 deaths

Figure 8: Impact of lock-down lifting and improved healthcare services on on COVID-19 deaths given individual behavior.

Figure 8 shows the COVID-19 related deaths given relaxation of COVID-19 restrictions and improvement of medical services. The red line in Figure 8a shows the impact of $100 \%$ lifting of lockdown interventions on dynamics of COVID-19 deaths in Kenya. If the government relaxed the lockdown measures by $50 \%$, then the dynamics of COVID-19 related death will be as depicted by the green line in Figure 8a. The red line in Figure 8b shows the impact of $100 \%$ lifting of lockdown interventions on dynamics of COVID-19 deaths in Kenya given change in individual behavior. If the government had lifted the lockdown measures by $50 \%$ in mid October 2020, then the dynamics of COVID-19 related death will be as depicted by the green line in Figure $8 \mathrm{~b}$ assuming individual change of behavior. On the other hand improving health-care services has an impact on the number of COVID-19 related deaths. The pink dotted line in Figure 8b shows the death dynamics when there is $100 \%$ restriction relaxation, assuming individual change of behavior and health-care services are improved. The COVID-19 death dynamics when health-care services are improved and $50 \%$ lockdown is applied is depicted by the dotted green line in the graph. From Figure 8b, there is no significant difference between relaxing the restriction measure completely or by $50 \%$ on COVID-19 related deaths assuming improved medical services and individual behavior change. Hence the government should strive to improve the health-care services/facilities before applying the intervention exit strategies.

\section{Discussions and Recommendations}

In this study, we applied the $S L I R D$ compartmental delay differential model to the daily reported cases of COVID-19 to estimate the transmission dynamics of COVID-19 and explore the influence of easing intervention measures by reopening schools and economy in Kenya. We evaluated the impact of individual behavior change with a society-wide relaxation of the partial lockdown measures and with aggressive targeted contact tracing and effective mass testing. We projected the number COVID-19 cumulative cases across different reopening strategies. 
The combination of the intervention measure put in place by the Kenyan Government have been effective in enhancing community-level social distancing and reducing the peak incidence of infections. In turn this has lessened the pressure on health services. The model results in our study has shown that, to reopen or restart the economy is a long-term process. It needs to be done sequentially. Going forward, a staggered reopening of businesses and learning institutions is highly recommended. If the Kenyan economy reopens in phases from October 2020, prevention of a second wave would require $30 \%$ of the business and learning institutions opened in October 2020, followed by an increase to $50 \%$ in April 2021. However, without such measures, reopening of the businesses and educational institutions together with unplanned relaxing of the partial lock-down measures are likely to induce a second pandemic wave.

From the model simulations, it is also shown that with aggressive targeted and effective mass testing, the asymptomatic and symptomatic patients who are not yet in hospital will be identified and either hospitalized or quarantined in isolation centres hence preventing further transmission. The asymptomatic persons are the problem and the contact tracing and mass testing should be targeting this population.

Before the government lifts COVID-19 restrictions, there is need for evidence that the virus is being suppressed and the government must ensure that there is robust public health capacity system in place. Evidence from the simulations points out that, return to normalcy will require change of individual behavior in following the disease intervention measures. Proper wearing of face masks and personal hygiene together with aggressive contact-tracing and active testing will not only prevent a second wave, but also likely to be able to control the transmission of COVID-19, hence preventing new cases and deaths.

The model presented in this paper is not without shortcomings. It is vital to note that as the epidemic evolves, new information has been coming with respect to the virus, intervention measures and issues related to vaccine development. It is thus important to adapt such a model to the changes in the disease landscape for better predictions. the model could also include vital dynamics especially if projected over a few years. The data availability with respect to the pandemic has been changing and this can also impact the fitting process and projections. Despite these shortcomings, the model presents some interesting results on the potential role of the different intervention measure taken by the Kenyan government.

\section{Acknowledgement}

The authors are very grateful to the anonymous reviewers for their careful reading and constructive comments.

\section{Funding}

The authors received no funding for this research work.

\section{Availability of data and materials}

All data generated or analysed in this study are included in this manuscript.

\section{Authors' contributions}

R.W.M produced the first draft of the manuscript. All authors read and approved the final manuscript. 


\section{Ethical approval and consent to participate}

Not applicable.

\section{Consent for publication}

Not applicable.

\section{Conflicts of Interest}

The authors declare that there is no conflict of interest regarding the publication of this article

\section{References}

[1] Sasmita Poudel Adhikari and et al. Epidemiology, causes, clinical manifestation and diagnosis, prevention and control of coronavirus disease (covid-19) during the early outbreak period: a scoping review. Infectious Diseases of Poverty, 9(1):1-12, 2020.

[2] Nanshan Chen, Min Zhou, and et al. Epidemiological and clinical characteristics of 99 cases of 2019 novel coronavirus pneumonia in Wuhan, China: a descriptive study. The Lancet, 395(10223):507-513, 2020.

[3] Tian-Mu Chen and et al. A mathematical model for simulating the phase-based transmissibility of a novel coronavirus. Infectious Diseases of Poverty, 9(1):24, 2020.

[4] Amirhoshang Hoseinpour Dehkordi and et al. Understanding epidemic data and statistics: A case study of covid-19. J Med Virol, pages 1-15, 2020.

[5] Odo Diekmann, Johan Andre Peter Heesterbeek, and Johan AJ Metz. On the definition and the computation of the basic reproduction ratio $\left(R_{0}\right)$ in models for infectious diseases in heterogeneous populations. Journal of Mathematical Biology, 28(4):365-382, 1990.

[6] Rachel T Esra and et al. Evaluating the impact of non-pharmaceutical interventions for SARSCoV-2 on a global scale. preprint, Infectious Diseases (except HIV/AIDS), August 2020.

[7] Joel Hellewell and et al. Feasibility of controlling COVID-19 outbreaks by isolation of cases and contacts. The Lancet Global Health, 4(8):e488-e496, 2020.

[8] Ying-Hen Hsieh. 2015 middle east respiratory syndrome coronavirus (mers-cov) nosocomial outbreak in South Korea: insights from modeling. PubMed, 3(e1505):1-9, 2015.

[9] Zhiliang Hu and et al. Clinical characteristics of 24 asymptomatic infections with covid-19 screened among close contacts in Nanjing, China. Science China Life Sciences, pages 1-6, 2020.

[10] Chaolin Huang and et al. Clinical features of patients infected with 2019 novel coronavirus in Wuhan, China. The Lancet, 395(10223):497-506, 2020.

[11] Matt J Keeling, T. Deirdre Hollingsworth, and Jonathan M Read. The Efficacy of Contact Tracing for the Containment of the 2019 Novel Coronavirus (COVID-19). preprint, Public and Global Health, February 2020. 
[12] Yunhwan Kim and et al. The characteristics of middle eastern respiratory syndrome coronavirus transmission dynamics in south korea. PubMed, 7(1):49-55, 2016.

[13] Olga Krylova and David J. D. Earn. Effects of the infectious period distribution on predicted transitions in childhood disease dynamics. Journal of The Royal Society Interface, 10(84):20130098, 2013.

[14] Adam J. Kucharski and et al. Early dynamics of transmission and control of COVID-19: a mathematical modelling study. The Lancet Infectious Diseases, 20:553-558, 2020.

[15] Abhishek Kumar and Nilam. Mathematical analysis of a delayed epidemic model with nonlinear incidence and treatment rates. Journal of Engineering Mathematics, 115(1):1-20, April 2019.

[16] Toshukazi Kuniya. Prediction of the epidemic peak of coronavirus disease in Japan. Journal of Clinical Medicine, 9(3)(789):1-7, 2020. https://doi.org/10.3390/jcm9030789.

[17] Qianying Lin and et al. A conceptual model for the outbreak of coronavirus disease 2019 (covid19) in Wuhan, China with individual reaction and governmental action. International Journal of Infectious Diseases, 93:211-216, 2020.

[18] Rachel Waema Mbogo and John W. Odhiambo. COVID-19 outbreak, social distancing and mass testing in Kenya - insights from a mathematical model. Afrika Matematika, 1:1-19, January 2021.

[19] Rachel Waema Mbogo and Titus O . Orwa. SARS-COV-2 outbreak and control in kenya- insights from a mathematical model. Infectious Disease Modelling, 1:1-16, January 2021.

[20] Samuel Mwalili, Mark Kimathi, Viona Ojiambo, Duncan Gathungu, and Rachel Mbogo. SEIR model for COVID-19 dynamics incorporating the environment and social distancing. BMC Research Notes, 13(352):1-5, December 2020.

[21] Faïçal Ndaïrou, Iván Area, Juan J. Nieto, and Delfim F. M. Torres. Mathematical modeling of COVID-19 transmission dynamics with a case study of Wuhan. Chaos, Solitons \& Fractals, 135:109846, 2020.

[22] Calistus N. Ngonghala and et al. Mathematical assessment of the impact of non-pharmaceutical interventions on curtailing the 2019 novel Coronavirus. Mathematical Biosciences, 325:1-27, 2020.

[23] Kenya Ministry of Health. Covid-19 situational reports. Press, 2020.

[24] Kenya Ministry of Health. Press statement on the update of the coronavirus in the country. Press, pages $1-3,2020$.

[25] John Ojal and et al. Revealing the extent of the COVID-19 pandemic in Kenya based on serological and PCR-test data. preprint, Epidemiology, September 2020.

[26] Jasmina Panovska-Griffiths. Can mathematical modelling solve the current covid-19 crisis? BMC Public Health, 20(551):1-3, 2020.

[27] Jasmina Panovska-Griffiths, Cliff Kerr, and et al. Determining the optimal strategy for reopening schools, work and society in the UK: balancing earlier opening and the impact of test and trace strategies with the risk of occurrence of a secondary COVID-19 pandemic wave. preprint, Infectious Diseases (except HIV/AIDS), June 2020.

[28] Kiesha Prem and et al. The effect of control strategies to reduce social mixing on outcomes of the COVID-19 epidemic in Wuhan, China: a modelling study. The Lancet Public Health, 5(5):e261e270, 2020. 
[29] G.P. Saha, S. Samanta and J.J. Nieto. Epidemic model of covid-19 outbreak by inducing behavioural response in population. Nonlinear Dyn, 102:455-487, January 2020.

[30] Sadoof Alaswad SNofe Al-Asuoad and Meir Shillor Libin Rong. Mathematical model and simulations of mers outbreak: Predictions and implications for control measures. Biomath, 5(2):1612141, January 2016.

[31] Pathum Sookaromdee and Viroj Wiwanitkit. Imported cases of 2019-novel coronavirus (2019ncov) infections in Thailand: Mathematical modelling of the outbreak. Asian Pacific Journal of Tropical Medicine, 13(3):139-140, 2020.

[32] Pauline Van den Driessche and James Watmough. Reproduction numbers and sub-threshold endemic equilibria for compartmental models of disease transmission. Mathematical Biosciences, 180(1-2):29-48, 2002.

[33] Robert Verity and et al. Estimates of the severity of coronavirus disease 2019: a model-based analysis. The Lancet Infectious Diseases, 20(1):669-677, 2020.

[34] WHO. Coronavirus 2019 (COVID-19): Dashboard. World Health Organization, 2020.

[35] WHO. Coronavirus disease 2019 (COVID-19): situation report, 51. World Health Organization, 2020 .

[36] WHO. World health organization. novel coronavirus situation report -2. january 22, 2020. World Health Organization, 2020.

[37] WHO. World health organization. Q \& A on coronaviruses (covid-19). World Health Organization, 2020.

[38] Po Yang and et al. Feasibility study of mitigation and suppression strategies for controlling covid-19 outbreaks in London and Wuhan. PloS one, 8(15):1-19, 2020.

[39] Zifeng Yang, Zhiqi Zeng, and et al. Modified SEIR and AI prediction of the epidemics trend of COVID-19 in China under public health interventions. Journal of Thoracic Disease, 12(3):165174, 2020.

[40] Dahai Zhao and et al. A comparative study on the clinical features of covid-19 pneumonia to other pneumonias. Clinical Infectious Diseases, 15(71):756-761, 2020.

[41] Xiang Zhou and et al. Forecasting the Worldwide Spread of COVID-19 based on Logistic Model and SEIR Model. preprint, BMJ Yale, 2020. 
Figures

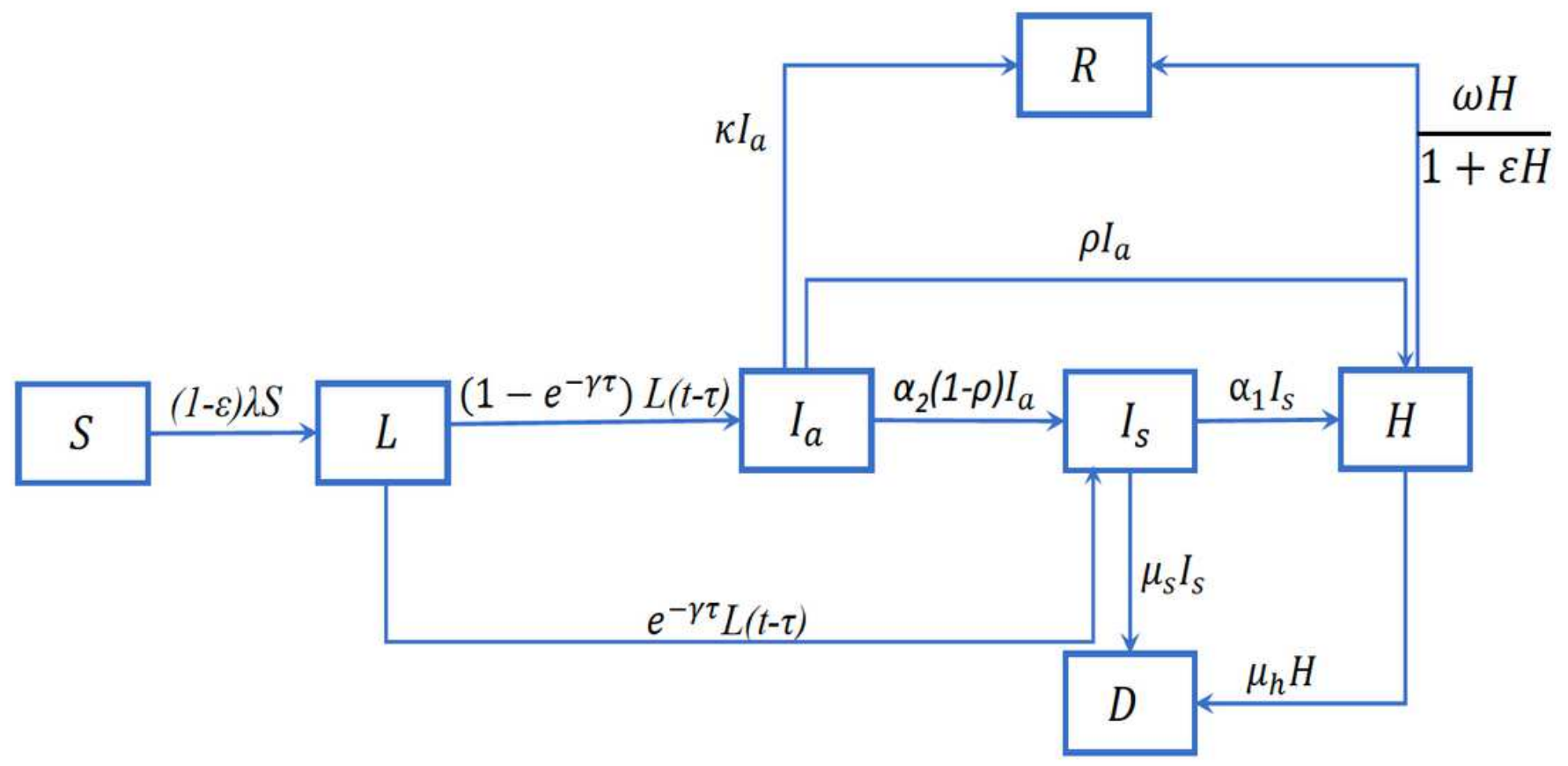

Figure 1

The model diagram 


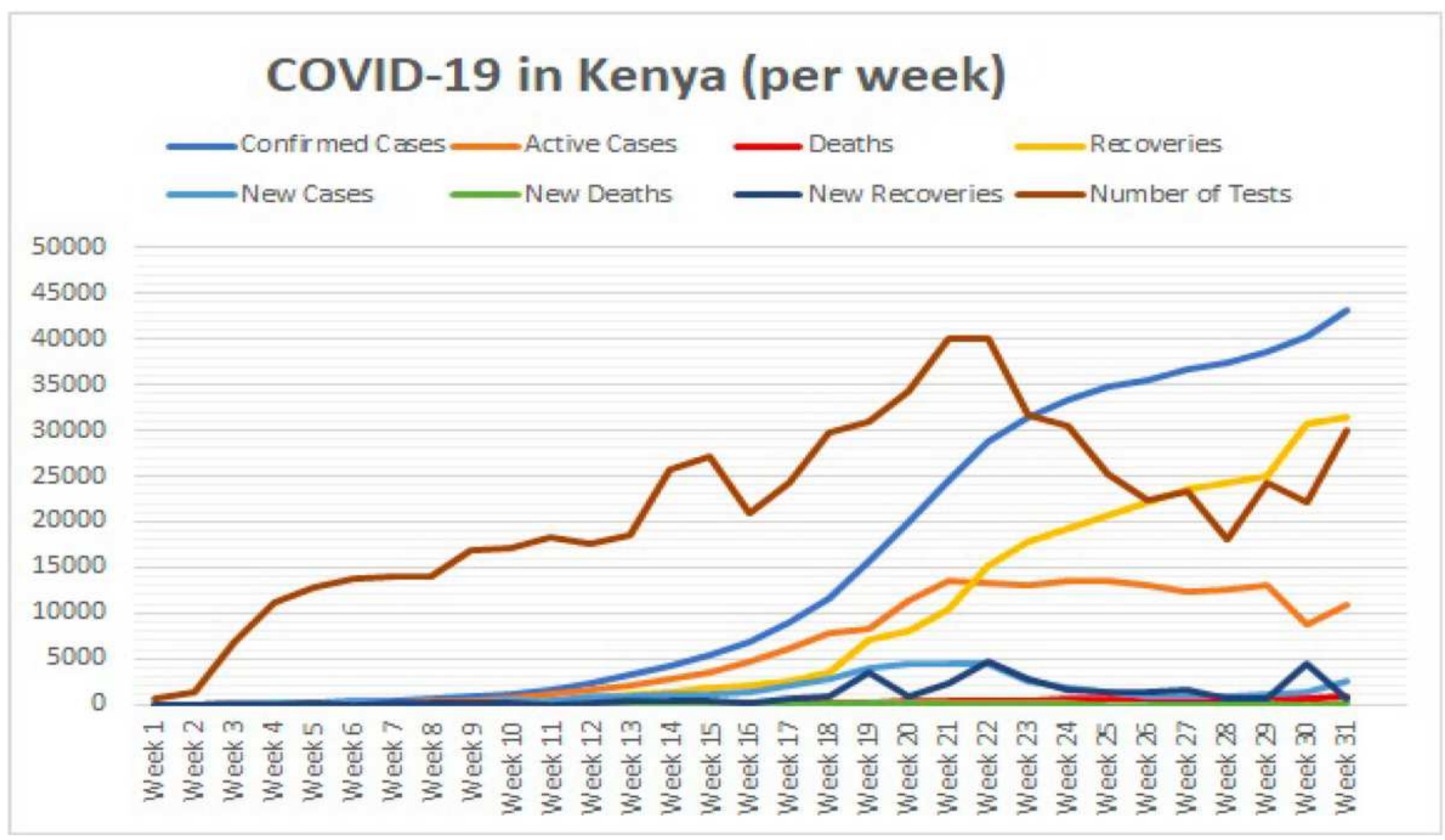

\section{Figure 2}

Weekly COVID-19 data for Kenya (March 13-October 15, 2020), for confirmed cases, active cases, deaths, recoveries, new cases, new deaths, new recoveries and the number of test done.

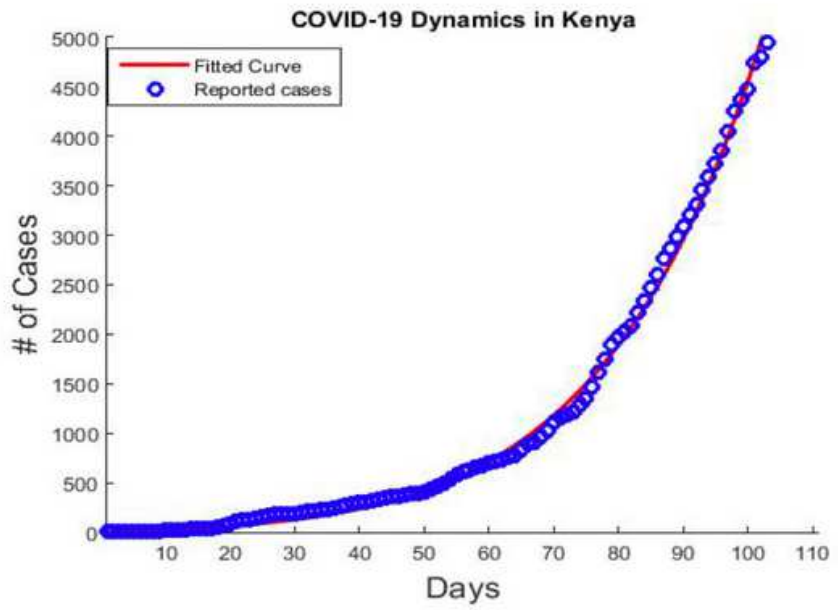

(a) COVID-19 cases with actual Kenyan Data

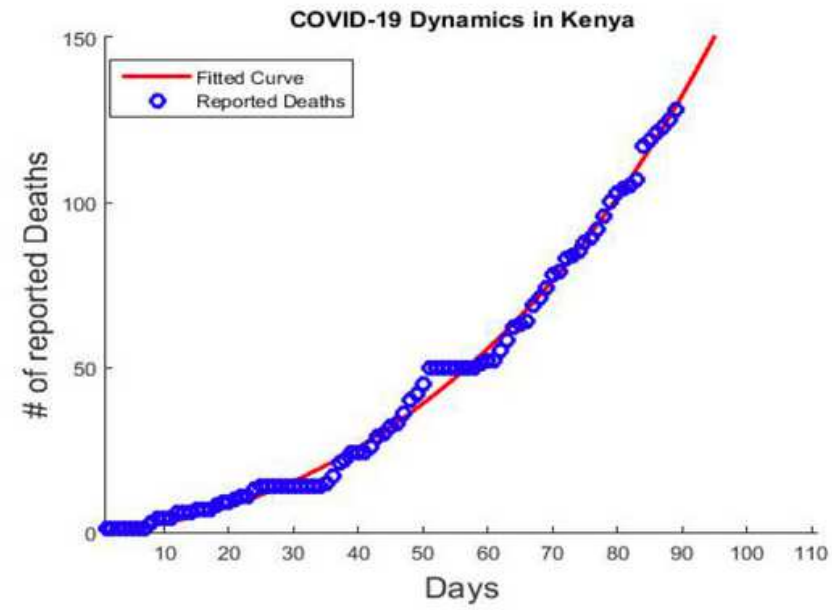

(b) COVID-19 Deaths with actual Kenyan Data

\section{Figure 3}

Curve fitting with reported Kenyan data 


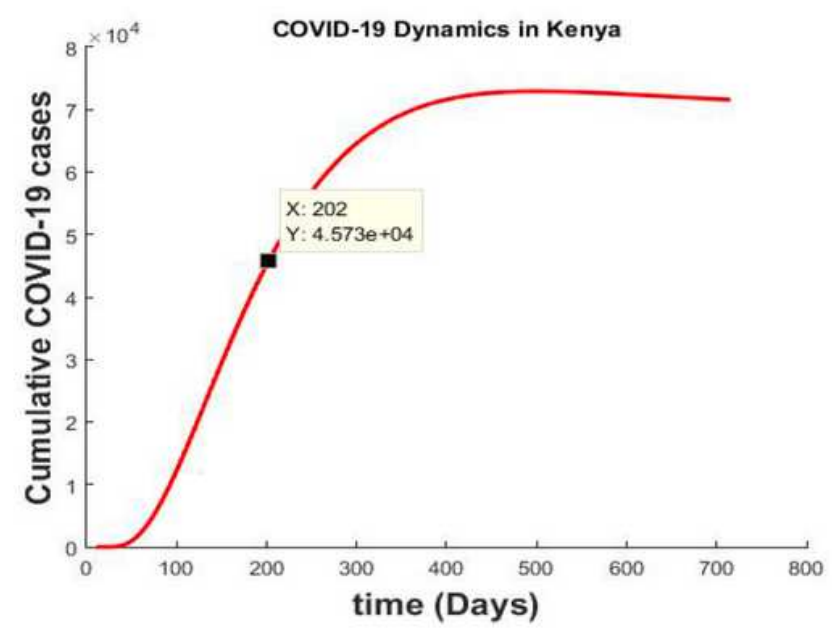

(a) COVID-19 projected cases in Kenya

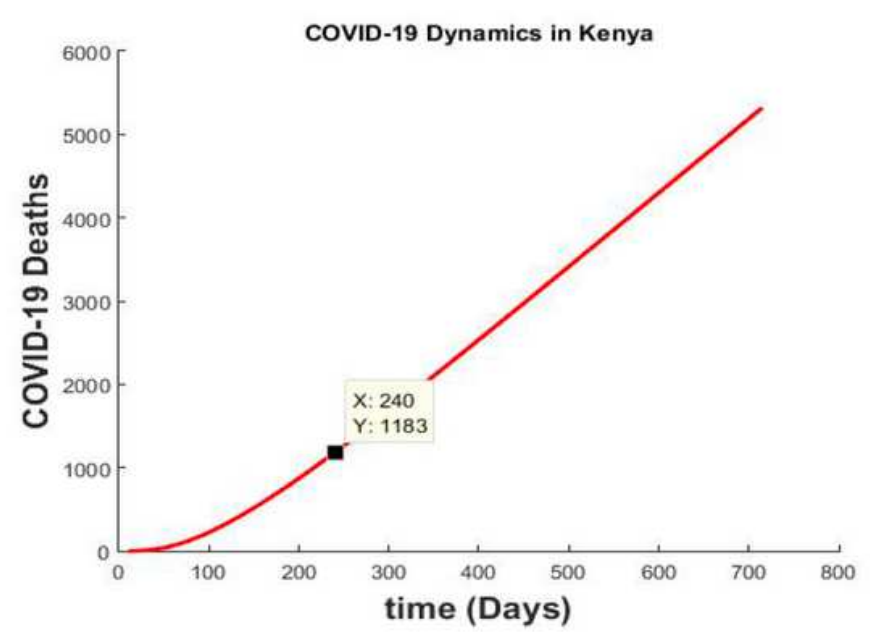

(b) COVID-19 projected Deaths in Kenya

\section{Figure 4}

Projections of COVID-19 Cumulative cases and number of deaths Kenya over a two year period

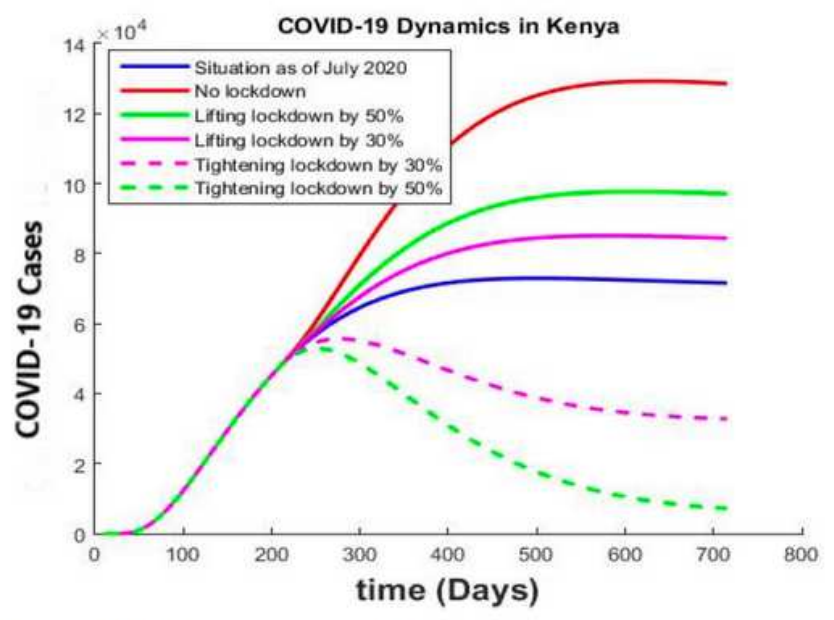

(a) COVID-19 dynamics with lifting of lock-down intervention measures

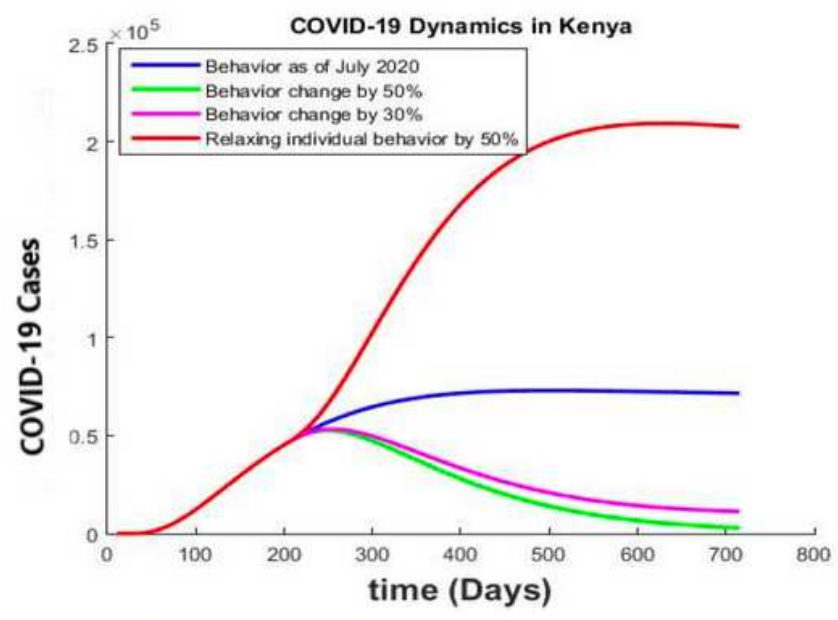

(b) COVID-19 dynamics with individual behavior change

\section{Figure 5}

Impact of individual behaviour change and lock-down lifting on cumulative COVID-19 cases. 


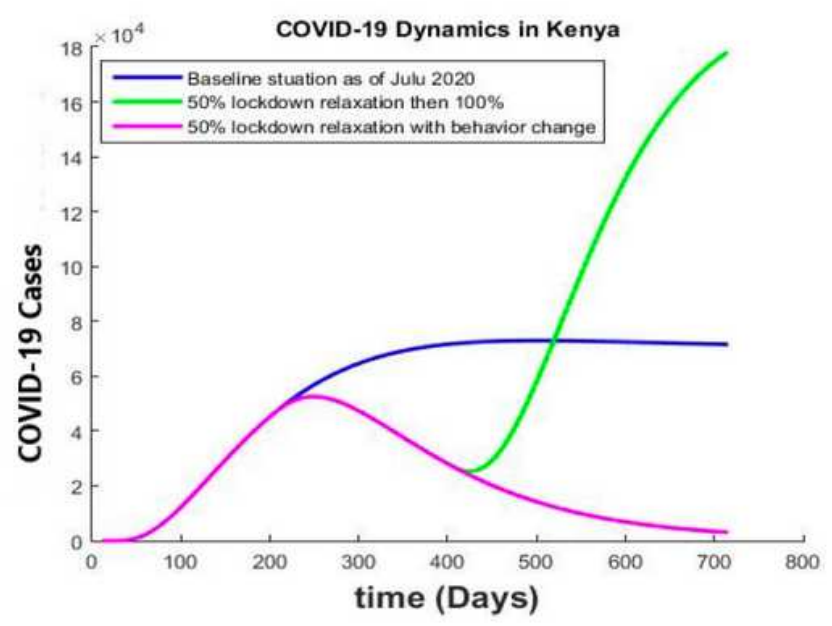

(a) COVID-19 dynamics with lockdown easing and individual behavior

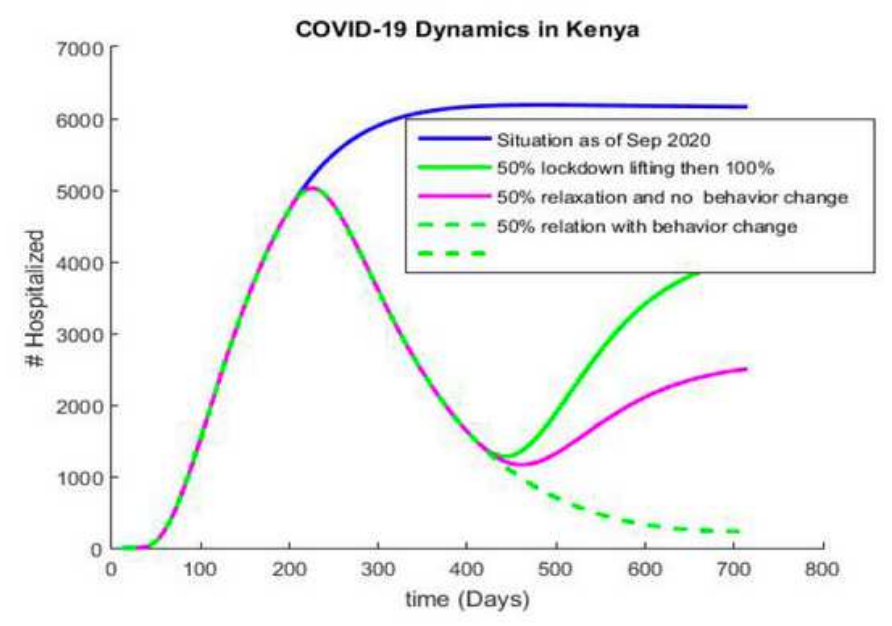

(b) Hospitalized cases with Lock-down easing and individual behavior

\section{Figure 6}

Impact of individual behaviour change and lock-down lifting on number of hospitalized cases.

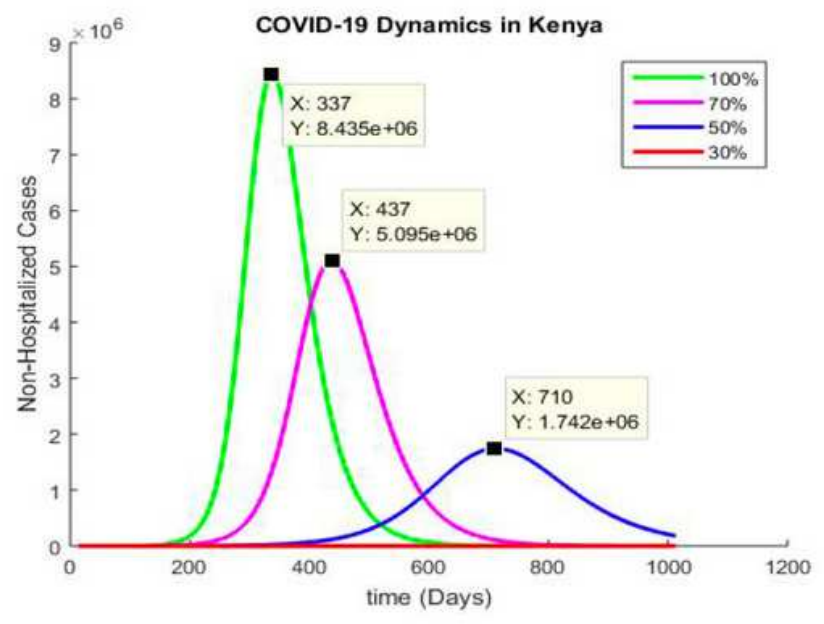

(a) Intervention relaxation impact on Nonhospitalized infected population

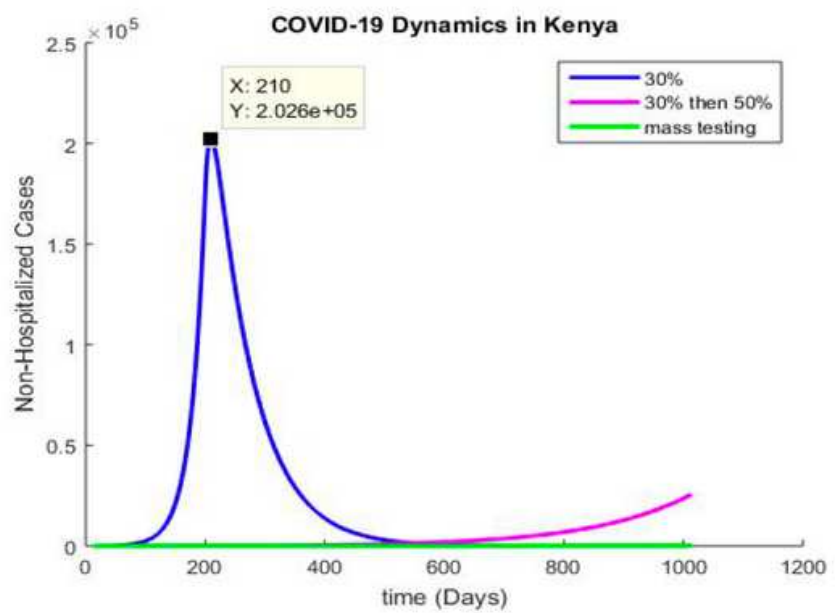

(b) Impact of aggressive mass testing on infected population

Figure 7

Impact of Mass testing and lock-down lifting on COVID-19 diffusion given individual behavior. 


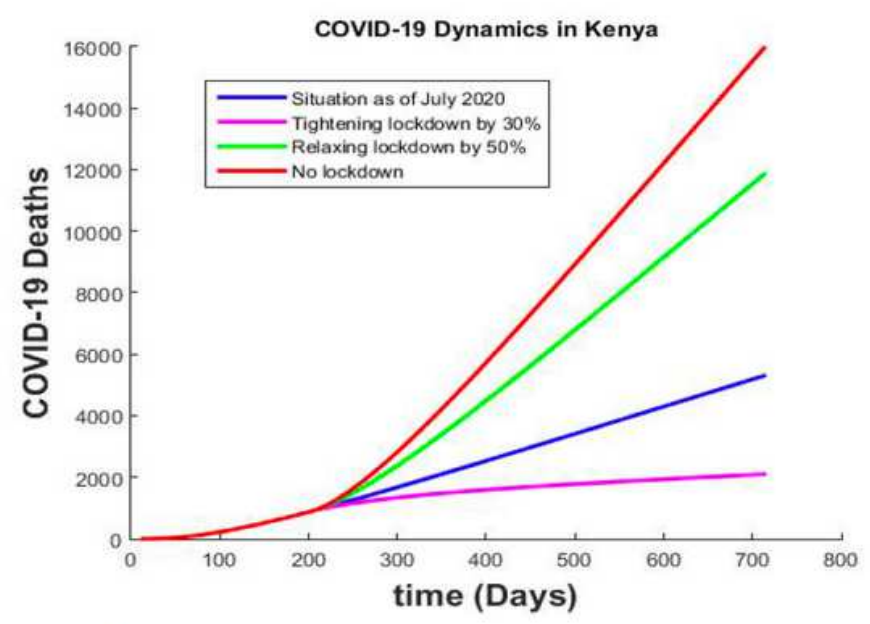

(a) The impact of individual behavior change on COVID-19 deaths

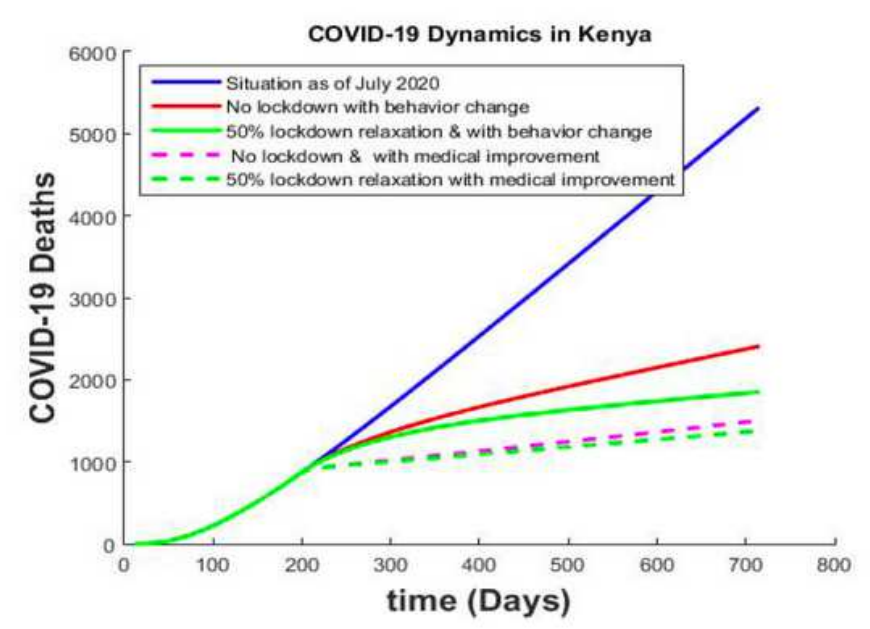

(b) The impact of improved healthcare services on COVID-19 deaths

\section{Figure 8}

Impact of lock-down lifting and improved healthcare services on on COVID-19 deaths given individual behavior. 